\section{Expression of B-cell activating factor, a proliferating inducing ligand and its receptors in primary central nervous system lymphoma}

\author{
Tobias Birnbaum,1 Sigrid Langer, 1 \\ Sigrun Roeber, ${ }^{2}$ Louisa von Baumgarten, 1 \\ Andreas Straube 1 \\ Departments of 1 Neurology and \\ 2Neuropathology, Ludwig-Maximilians \\ University, Munich, Germany
}

\section{Abstract}

B-cell activating factor belonging to the tumor necrosis factor family (BAFF) and a proliferating inducing ligand (APRIL) might play an important role in the pathogenesis of systemic B-cell malignancies. However, the BAFF/APRIL system has not been systematically evaluated in primary central nervous system lymphoma (PCNSL) to date. We assessed the expression of BAFF, APRIL and its receptors BAFF-R (BAFF receptor), BCMA (B-cell maturation antigen) and TACI (transmembrane activator and calcium modulator cyclophilin ligand interactor) in five PCNSL specimens by immunohistochemical staining. We found extensive expression of BAFF and weak to moderate expression of APRIL, BAFF-R, BCMA, and TACI in all specimens. CD20 positive cells showed expression of both ligands and receptors at the same time. Our results indicate that autocrine stimulation of the BAFF/APRIL system might be involved in the pathogenesis of PCNSL.

\section{Introduction}

Primary central nervous system lymphoma (PCNSL) is a rare variant of extranodal nonHodgkins lymphoma (NHL). Cure is achieved only in a minority of patients using high-dose chemotherapy with or without whole brain irradiation. ${ }^{1}$ However, combined chemoradiotherapy often is associated with severe neurotoxicity leading to dementia. Therefore, new treatment targets are urgently needed.

Little is known about the pathogenesis of PCNSL. Increased expression of nuclear factor kappa-B (NF-kB)-regulated genes has been detected suggesting that this signaling pathway might be of particular importance. ${ }^{2,3}$ B-cell activating factor belonging to the tumor necrosis factor family (BAFF) and a proliferating inducing ligand (APRIL) physiologically stimulate survival of B-cells through activation of the NF-kB-pathway. ${ }^{4}$ BAFF is usually expressed by macrophages, monocytes, and T-, but not Bcells. It binds to three receptors: BAFF receptor (BAFF-R), B-cell maturation antigen (BCMA) and transmembrane activator and calcium modulator cyclophilin ligand interactor (TACI). APRIL binds to BCMA and TACI. The BAFF/APRIL system might play an important role in the pathogenesis of systemic B-cell malignancies.4-7 However, this signaling pathway has not been systematically evaluated in PCNSL to date. Aim of this study was to assess the expression profile of the BAFF/APRIL system in PCNSL.

\section{Materials and Methods}

Formalin-fixed, paraffin-embedded tissue sections from biopsy specimens of five newly diagnosed PCNSL were assessed. Antigen retrieval was done with target retrieval solution (S1699, Dako, Germany) for $10 \mathrm{~min}$ at $98.5^{\circ} \mathrm{C}$. Endogenous peroxidase activity was reduced by incubation with $3 \% \mathrm{H} 202$ for $10 \mathrm{~min}$. We used the following primary antibodies: rabbit antihuman BAFF (ab117256, dilution 1:12.5), goat anti-human APRIL (ab110848, dilution 1:100), rabbit anti-human BAFF-R (ab5965, dilution 1:100), rabbit anti-human BCMA (ab115315, dilution 1:50), rabbit anti-human TACI (ab79023, dilution 1:50), mouse anti-human CD20 [ab9475 (L26), dilution 1:35], rabbit antihuman CD3 (ab5690, dilution 1:100) (all ABCAM, Cambridge, MA, USA), mouse antihuman CD68 (PG-M1, M 0876, dilution 1:100, Dako, Glostrup, Denmark) and rabbit antihuman BAFF (ABIN740175, dilution 1:50, antibodies-online inc., Atlanta, GA, USA). The following secondary antibodies were used: horseradish peroxidase conjugated polyclonal goat anti-rabbit (dilution 1:100), horseradish peroxidase conjugated polyclonal goat anti-mouse (dilution 1:200, both Dako, Glostrup, Denmark), and horseradish peroxidase conjugated polyclonal donkey anti-goat (dilution 1:125, Dianova, Germany). Visualization was done with DAB Chromogen (Dako, Glostrup, Denmark). Haematoxylin was used for counterstaining. The ethics committee of the University of Munich approved the study.

\section{Results}

We evaluated tissue sections from five patients with newly-diagnosed PCNSL. All tumors were histologically classified as diffuse large B-cell lymphoma (DLBCL). Immunohistochemistry revealed extensive nuclear expression of BAFF in all specimens (Table 1, Figure 1). BAFF staining was validat-
Correspondence: Tobias Birnbaum, Department of Neurology, Ludwig-Maximilians University, Marchioninistr. 15, 81377 Munich, Germany. Tel. +49.8970950 - Fax: +49.8951603677.

E-mail: tobias.birnbaum@med.uni-muenchen.de

Key words: PCNSL; BAFF; APRIL; TACI; BCMA

Conflict of interests: the authors report no potential conflict of interests.

Contributions: TB, SL, AS, were responsible for the conception of the study, analysis and interpretation of data; TB, wrote the manuscript; LvB, SL, SR, were responsible for immunohistochemical staining, acquisition of photographs and preparing figures.

Received for publication: 14 September 2012. Revision received: 12 February 2013.

Accepted for publication: 25 February 2013.

This work is licensed under a Creative Commons Attribution NonCommercial 3.0 License (CC BYNC 3.0).

CC Copyright T. Birnbaum et al., 2013

Licensee PAGEPress, Italy

Neurology International 2013; 5:e4

doi:10.4081/ni.2013.e4

ed using two different antibodies. In contrast, APRIL was expressed in variable amounts in 4/5 specimens. Expression of BAFF and APRIL was not restricted to stromal or inflammatory cells, but was found mainly in CD20 positive tumor cells. Furthermore, all tumors showed at least weak expression of all three receptors (Table 1, Figure 1). Two tumors showed extensive expression of TACI and weak to moderate expression of BCMA and BAFF-R. The remaining specimens showed weak to moderate expression of all three receptors. As for the ligands, expression of BAFF-R, BCMA and TACI was found mainly within CD20 positive cells. All in all, most CD20 positive cells expressed not only BAFF/APRIL, but also at least one corresponding receptor.

\section{Discussion and Conclusions}

Expression of components of the BAFF/APRIL signaling pathway has been shown in various systemic lymphomas.4-8 Overexpression of APRIL has been correlated with higher aggressiveness of B-cell lymphomas and expression of TACI has been associated with poor prognosis in Burkitt lymphoma..$^{6,9}$ Another study suggested that BAFF is able to decrease apoptosis and to enhance survival of malignant B-cells. ${ }^{8}$ Furthermore, patients with increased serum BAFF levels had 
a worse prognosis and BAFF levels correlated with transformation to a more aggressive tumor phenotype. ${ }^{8}$

So far, only one report deals with this signaling pathway in PCNSL: Krumbholz et al. found expression of mRNA for BAFF and its receptors in five PCNSL specimens. Evaluation of expression of the corresponding proteins, however, was done only for BAFF-R by immunohistochemistry. 10 The authors assumed that BAFF might be

Table 1. Semi-quantitative assessment of expression of the BAFF/APRIL system in the different tumor specimens.

\begin{tabular}{lccccc} 
PCNSL & BAFF & APRI & BAFF-R & BCMA & TACI \\
E123 & +++ & ++ & + & ++ & +++ \\
E236 & +++ & ++ & ++ & ++ & ++ \\
\hline E370 & +++ & + & ++ & + & +++ \\
E582 & +++ & - & + & + & + \\
\hline E753 & +++ & + & + & + & ++ \\
\hline
\end{tabular}

PCNSL, primary central nervous system lymphoma; BAFF, B-cell activating factor belonging to the tumor necrosis factor family; APRIL, a proliferating inducing ligand; BAFF-R, BAFF receptor; BCMA, B-cell maturation antigen; TACI, transmembrane activator and calcium modulator cyclophilin ligand interactor.

- negative; $+<30 \%$ positive cells $;++30-60 \%$ positive cells $;+++>60 \%$ positive cells.
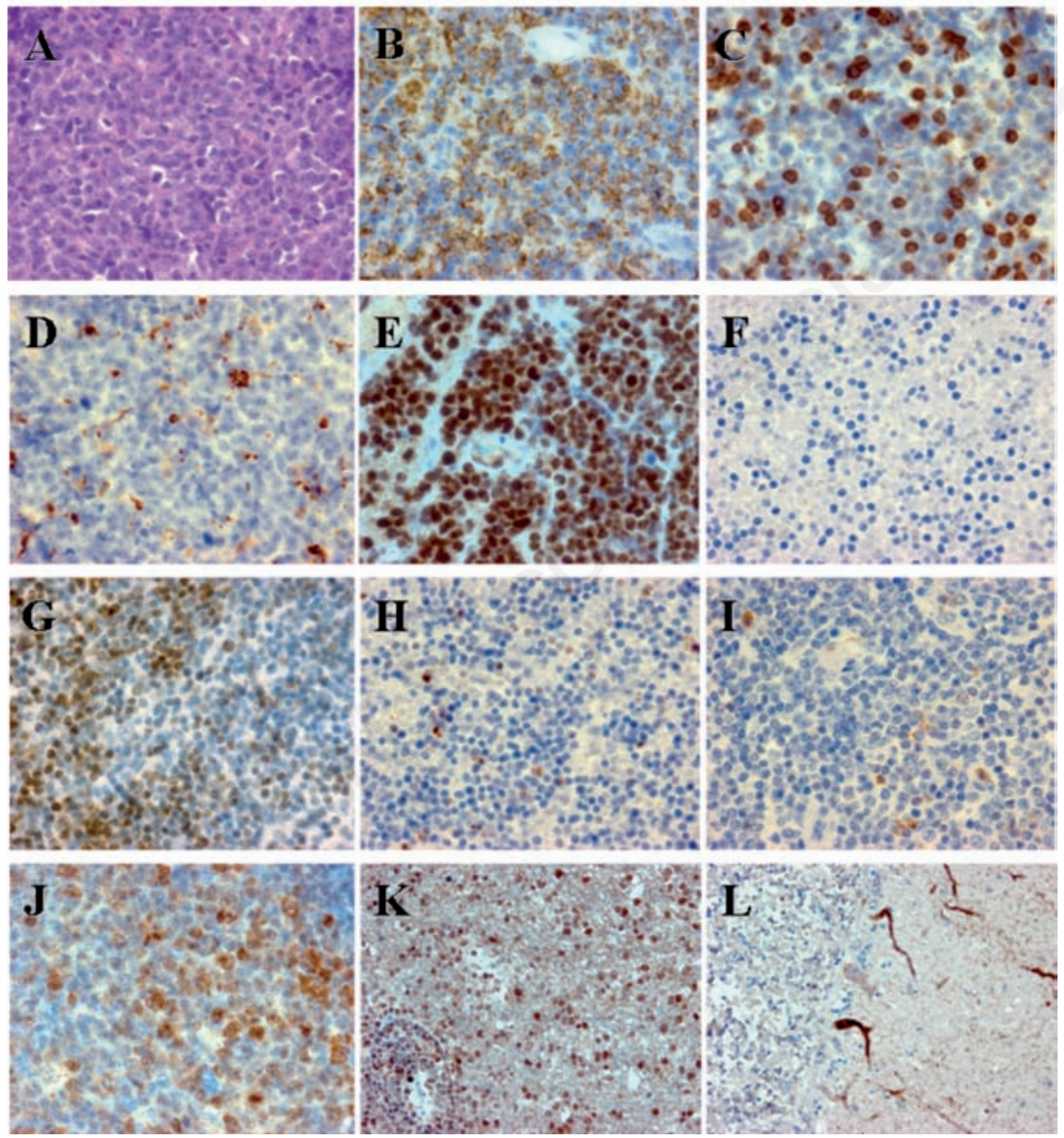

Figure 1. Immunohistochemical stainings of specimen E582: Haematoxylin \& Eosin staining (A) shows densely packed lymphoid tumor cells, which are positive for CD20 (B). Reactive CD3 positive T cells $(\mathrm{C})$ and CD68 positive microglial cells $(\mathrm{D})$ are found within the tumor mass. CD20 positive tumor cells show extensive expression of BAFF (E) and weak to moderate expression of BAFF-R (G), BCMA (H) and TACI (I). No expression of APRIL (F) was found in this specimen. Tonsil tissue served as positive control of anti-BAFF staining (J). Validation of anti-BAFF staining using a second antibody in tumor specimen E236 (K). Cerebellar tissue was used as positive control of anti-APRIL staining (L). Original magnification $\times 200(\mathrm{~K}, \mathrm{~L}), \times 400(\mathrm{~A}-\mathrm{J})$.

expressed by astrocytes since they had found BAFF-expressing astrocytes in the context of multiple sclerosis lesions. In contrast, our results show for the first time, that CD20 positive tumor cells themselves extensively produce BAFF. Therefore, rather autocrine than paracrine stimulation of this signaling pathway might promote proliferation of lymphoma cells. These results indicate a pathogenic role of the BAFF/APRIL system not only in systemic B-cell lymphomas, but also in PCNSL. Therefore, this signaling pathway might represent a new treatment target. Indeed, Lyu et al. found a significant antitumor activity of a BAFF fusion toxin in DLBCL cell lines, which expressed BAFF receptors. ${ }^{11}$ Other groups recently achieved promising results using BAFF-mediated targeting of liposomal vincristine to malignant B-cells or monoclonal antibodies directed against APRIL. ${ }^{12,13}$

Further in vitro and in vivo studies should address the role of the BAFF/APRIL system in PCNSL and analyze whether blocking this signaling pathway might be beneficial in the treatment of this disease.

\section{References}

1. Roth P, Korfel A, Martus P, Weller M. Pathogenesis and management of primary CNS lymphoma. Expert Rev Anticancer Ther 2012;12:623-33.

2. Courts C, Montesinos-Rongen M, MartinSubero JI, et al. Transcriptional profiling of the nuclear factor-kappaB pathway identifies a subgroup of primary lymphoma of the central nervous system with low BCL10 expression. J Neuropathol Exp Neurol 2007;66:230-7.

3. Montesinos-Rongen M, Schmitz R, Brunn A, et al. Mutations of CARD11 but not TNFAIP3 may activate the NF-kappaB pathway in primary CNS lymphoma. Acta Neuropathol 2010;120:529-35.

4. Mackay F, Tangye SG. The role of the BAFF/APRIL system in B cell homeostasis and lymphoid cancers. Curr Opin Pharmacol 2004;4:347-54.

5. Wada K, Maeda K, Tajima K, et al. Expression of BAFF-R and TACI in reactive lymphoid tissues and B-cell lymphomas. Histopathology 2009;54:221-32.

6. Moreaux J, Veyrune JL, De Vos J, Klein B. APRIL is overexpressed in cancer: link with tumor progression. BMC Cancer 2009;9:83.

7. Rodig SJ, Shahsafaei A, Li B, et al. BAFF-R, the major B cell-activating factor receptor, is expressed on most mature $\mathrm{B}$ cells and $\mathrm{B}$ cell lymphoproliferative disorders. Hum Pathol 2005;36:1113-9.

8. Novak AJ, Grote DM, Stenson M, et al. Expression of BLyS and its receptors in B- 
cell non-Hodgkin lymphoma: correlation with disease activity and patient outcome. Blood 2004;104:2247-53.

9. Schwaller J, Schneider P, MhawechFauceglia P, et al. Neutrophil derived APRIL concentrated in tumor lesions by proteoglycans correlates with human Bcell lymphoma aggressiveness. Blood 2007;109:331-8.
10. Krumbholz M, Theil D, Derfuss T, et al. BAFF is produced by astrocytes and up-regulated in multiple sclerosis lesions and primary central nervous system lymphoma. J Exp Med 2005;201:195-200.

11. Lyu MA, Rai D, Ahn KS, et al. The rGel/BLyS fusion toxin inhibits diffuse large B-cell lymphoma growth in vitro and in vivo. Neoplasia 2010;12:366-75.
12. Zhang L, Gao H, Chen L, et al. Tumor targeting of vincristine by mBAFF-modified PEG liposomes in B lymphoma cells. Cancer Lett 2008;269:26-36.

13. Guadagnoli M, Kimberley FC, Phan U, et al. Development and characterization of APRIL antagonistic monoclonal antibodies for treatment of B-cell lymphomas. Blood 2011;117:6856-65. 